\title{
Inhibition of TGF- $\beta$ signaling with halofuginone can enhance the antitumor effect of irradiation in Lewis lung cancer
}

\author{
This article was published in the following Dove Press journal: \\ OncoTargets and Therapy \\ 2 December 2015 \\ Number of times this article has been viewed
}

\section{Runlong Lin* \\ Shuai Yi* \\ Linlin Gong \\ Weishuai Liu \\ Peng Wang \\ Ningbo Liu \\ Lujun Zhao \\ Ping Wang}

Department of Radiation Oncology, Tianjin Medical University Cancer Institute and Hospital, National Clinical Research Center of Cancer, Key Laboratory of Cancer Prevention and Therapy, Tianjin, People's Republic of China

*These authors contributed equally to this work

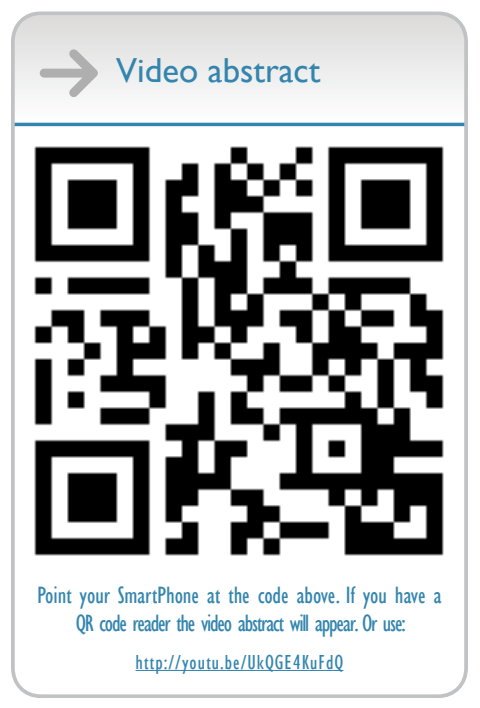

Correspondence: Lujun Zhao

Department of Radiation Oncology, Tianjin Medical University Cancer Institute and Hospital, Huan-hu-xi Road, He-xi District,

Tianjin 300060, People's Republic of China

Tel +862223340123

Fax +86222334 I 405

Email zhaolujun@tjmuch.com
Purpose: It was reported that halofuginone has inhibitory effects on transforming growth factor-beta (TGF- $\beta$ ) signaling pathway. The study was aimed to: 1) evaluate the antitumor effects of halofuginone in combination with radiation therapy; and 2) preliminarily explore the possible mechanisms associated with these effects.

Materials and methods: Lewis lung cancer (LLC) cell lines and xenograft model mice randomly received ionizing radiation, halofuginone, or combination treatment. The changes associated with antitumor effect of halofuginone, including hepatic and pulmonary metastases and survival, were observed. The migratory and invasive capabilities of LLC cells were investigated by using scratch assay and transwell chamber assay. The expression level of TGF- $\beta$ and its activation were assessed with enzyme-linked immunosorbent assay, immunohistochemistry, and Western blotting. Chi-square test and survival analysis were performed for statistical analysis. $P<0.05$ was regarded as statistically significant. Unless otherwise specified, data were expressed as mean \pm standard deviation $(\bar{x} \pm s)$.

Results: After irradiation, the migratory and invasive capabilities of LLC cells were strengthened, and the TGF- $\beta$ pathway was activated. The addition of halofuginone can significantly inhibit the migratory and invasive trend induced by irradiation, and the TGF- $\beta$ pathway was also inhibited. In animal xenograft model, the addition of halofuginone to irradiation inhibited the growth of subcutaneously implanted xenografts, reduced hepatic and pulmonary metastases, and improved survival of the mice. The effect was accompanied by a decrease in TGF- $\beta$ levels. In addition, halofuginone inhibited type I collagen expression and angiopoiesis.

Conclusion: Halofuginone treatment not only produces significant radiation-sensitizing effects but also inhibits hepatic and pulmonary metastases. The underlying mechanisms of these phenomena warrant additional studies.

Keywords: halofuginone, transforming growth factor- $\beta 1$, radiation therapy, lung cancer

\section{Introduction}

The transforming growth factor- $\beta$ (TGF- $\beta$ ) signaling pathway regulates tumor initiation, invasion, and metastasis. ${ }^{1}$ TGF- $\beta$ can promote tumor progression through angiopoiesis, suppression of immune surveillance, promotion of epithelial to mesenchymal transition, and degradation of extracellular matrix..$^{2-5}$ Radiation therapy is one of the most important treatment approaches for lung cancer. ${ }^{6}$ However, it is well established that radiation treatment can upregulate TGF- $\beta$ expression. ${ }^{7}$ Increases in TGF- $\beta$ levels may have detrimental effects on the control of lung cancer. ${ }^{8}$ In a mouse model, halofuginone can inhibit the TGF- $\beta$ signaling pathway and reduce radiation-induced fibrosis and contraction. ${ }^{9}$ Thus, we hypothesized that halofuginone could enhance the therapeutic effects of radiation treatment in lung cancer. A pilot 
study was performed to test this hypothesis and to explore the underlying mechanisms of any observed halofuginoneinduced effects; this study used Lewis lung cancer (LLC) cells and an animal xenograft model established with LLC cells in C57BL/6J mice.

\section{Materials and methods Reagents}

Halofuginone was obtained from Chifei Chemical Co., Ltd (Wuhan, Hubei, People's Republic of China). Working solutions for injection of halofuginone $(1,2.5$, or $5 \mu \mathrm{g} / 100 \mu \mathrm{L}$ ) were made each day by diluting the stock solution of halofuginone with $0.9 \% \mathrm{NaCl}$. The working solution was used within 1 hour of preparation. Sirius Red staining kit was obtained from Jiemei Gene Medicine Science and Technology Co., Ltd (Shanghai, People's Republic of China). Mouse monoclonal anti-mice TGF- $\beta 1$ and CD34 antibodies were obtained from Abcam Company (Cambridge, MA, USA). TGF- $\beta 1$ ELISA kit was obtained from R\&D Systems (Minneapolis, MN, USA).

\section{Cell culture}

LLC cell line was obtained from BioHermes Biomedical Science and Technology Co., Ltd (Wuxi, Jiangsu, People's Republic of China). Cells were thawed, cultured in Dulbecco's modified Eagle's medium (Thermo Fisher Scientific, Waltham, MA, USA), and supplemented with $10 \%$ fetal calf serum (Thermo Fisher Scientific) at $37^{\circ} \mathrm{C}$ in the presence of $5 \% \mathrm{CO}_{2}$-balanced air.

\section{LLC animal xenografts}

Animal welfare and experimental procedures were conducted in accordance with the guidelines for the care and use of laboratory animals (Ministry of Science and Technology of China, Beijing, People's Republic of China), and were approved by the animal ethics committee of Tianjin Medical University Cancer Institute and Hospital. C57BL/6J female mice were obtained from Weitonglihua Laboratory Animal Technology Co., Ltd. (Beijing, People's Republic of China). Suspensions of LLC cells were subcutaneously injected into the right hind limb of each mouse at a concentration of $6 \times 10^{6}$ cells/injection site. A balance and calipers were used to weigh mice and determine tumor sizes on alternate days, starting on the date of LLC cell inoculation. Tumor volumes were calculated using the formula volume $=$ $a \times b^{2} \times 0.4$, where $a$ and $b$ represent the longest and shortest tumor diameters, respectively. ${ }^{10}$ Mice were euthanized at scheduled times after treatment, and their tumors were harvested for analysis.

\section{Cell studies}

The toxicity of halofuginone on LLC was observed by the MTT method, and the appropriate dose of halofuginone was then determined before starting the experiments. LLC lines were divided into four groups: 1) control group, without any treatment; 2) halofuginone group, with management of cell culture with halofuginone $(30 \mathrm{ng} / \mathrm{mL})$ for 24 hours; 3 ) irradiation group, radiation was given using a $6 \mathrm{MV} \mathrm{X}$-ray irradiator (VARIAN 600CD) at a dose rate of $3 \mathrm{~Gy} /$ minute and a single dose of $10 \mathrm{~Gy}$; 4) combination group, with halofuginone administration as in the halofuginone group and radiation given as in the irradiation group.

\section{Animal studies}

A total of 36 model animals were randomly divided into the following four groups after the longest diameter of the subcutaneous tumor had attained a size of $0.5 \mathrm{~cm}: 1$ ) the control group, which received $0.9 \% \mathrm{NaCl}$ vehicle solution by gavage and sham irradiation; 2) the halofuginone group, which received halofuginone and sham irradiation; 3) the irradiation group, which received 30 Gy of radiation in 10 fractions and vehicle solution by gavage 1 hour prior to each radiation treatment; and 4) the combination group, which received halofuginone by gavage 1 hour prior to each radiation treatment.

The drug toxicity of halofuginone was evaluated before starting the experiments. Halofuginone dose of $1.0 \mu \mathrm{g} /$ day and $2.5 \mu \mathrm{g} /$ day had no effect on mouse physiology after 3 weeks of observation for drug toxicity, but one mouse died with a dosage of $5.0 \mu \mathrm{g} / \mathrm{day}$, and other mice were unresponsive. The dose of halofuginone used in this experiment was $2.5 \mu \mathrm{g} / \mathrm{day}$.

Selective irradiation of the right leg was accomplished by placing each unanesthetized animal into a specially built Lucite jig that immobilized the animal and facilitated ease of extension of the right hind leg into the radiation field. Lead shields designed as a part of the Lucite jigs assured that only the right hind limb of the immobilized animal was irradiated. A daily radiation dose of 3 Gy was delivered to the right hind leg of the animal by a $6 \mathrm{MV} \mathrm{X}$-ray irradiator (VARIAN $600 \mathrm{CD}$ ) at a dose rate of $3 \mathrm{~Gy} /$ minute for the following 10 days (source skin distance $=100 \mathrm{~cm}$, field size $=10 \mathrm{~cm}$ $\times 20 \mathrm{~cm}$ ). The mice that received sham irradiation received the same immobilization procedure but with no irradiation.

Immediately after irradiation, the animals were removed from the Lucite jig and housed in a climate and light/ dark-controlled environment and allowed free access to food and water. Three days after the treatment period, four randomly selected mice from each group were euthanized, and their tumor specimens were collected for further analysis. Hepatic and pulmonary metastases were also evaluated in gross specimens 
after mice were euthanized. Survival time was estimated from the implantation of tumor cells to the day of death. Figure 1 presents tumor models and treatment schematics.

\section{Immunohistochemistry}

Immunohistochemistry (IHC) was used to assess TGF- $\beta 1$ expression in xenografts. Briefly, $4 \mu \mathrm{m}$ paraffin-embedded sections were cut and stained to detect the presence of anti-TGF- $\beta 1$ monoclonal or other related antibody. After 12 hours, slides were rinsed in phosphate-buffered saline (PBS) and incubated with the second antibody for 40 minutes. Revelation was performed with diaminobenzidine reagent and hematoxylin counterstaining. Staining intensity was scored according to the following criteria: 0 for negative staining, 1 for weak staining, 2 for intermediate staining, and 3 for strong staining. The proportion of cells with positive staining was scored as 0 for none, 1 for $<1 / 3,2$ for $1 / 3-2 / 3$, and 3 for $>2 / 3$. The final staining score was the product of the intensity and proportion scores. ${ }^{11}$ Five high-power fields were evaluated on each slide. To further estimate TGF- $\beta 1$ levels in tumor tissue, enzyme-linked immunosorbent assay (ELISA) was performed using TGF- $\beta 1$ ELISA kits purchased from R\&D Systems.
Microvessel density (MVD) in xenograft tissue was assessed by anti-CD34 IHC. A single CD34-positive cell or cluster of endothelial cells that was clearly separate from adjacent microvessels and other connective tissue elements was regarded as a vessel. Large vessels with thick muscular walls and vessels in areas of sclerosis, tumor necrosis, nontumor populations, and benign lesions were excluded from vessel counts. Low-power light microscopy (at 10×10 magnification) was used to scan stained sections to identify regions with the highest vascular density. Vessels were then counted in ten regions at $10 \times 40$ magnification, and the numbers of microvessels in each region were recorded. The MVD was defined as the average value of the three densest regions.

\section{ELISA analysis}

To further estimate TGF- $\beta 1$ levels in LLC cells and tumor tissue, ELISA was performed using TGF- $\beta 1$ ELISA kits purchased from R\&D Systems. The biotinylated antibodies were added and incubated overnight at $4^{\circ} \mathrm{C}$. Then the samples with total protein were incubated in ELISA plates for 60 minutes at $37^{\circ} \mathrm{C}$. After washing with PBS, the cells were allowed to react with horseradish peroxidase-avidin for 30 minutes at $37^{\circ} \mathrm{C}$. The chromogen $3,3^{\prime}, 5,5^{\prime}$-tetramethylbenzidine was

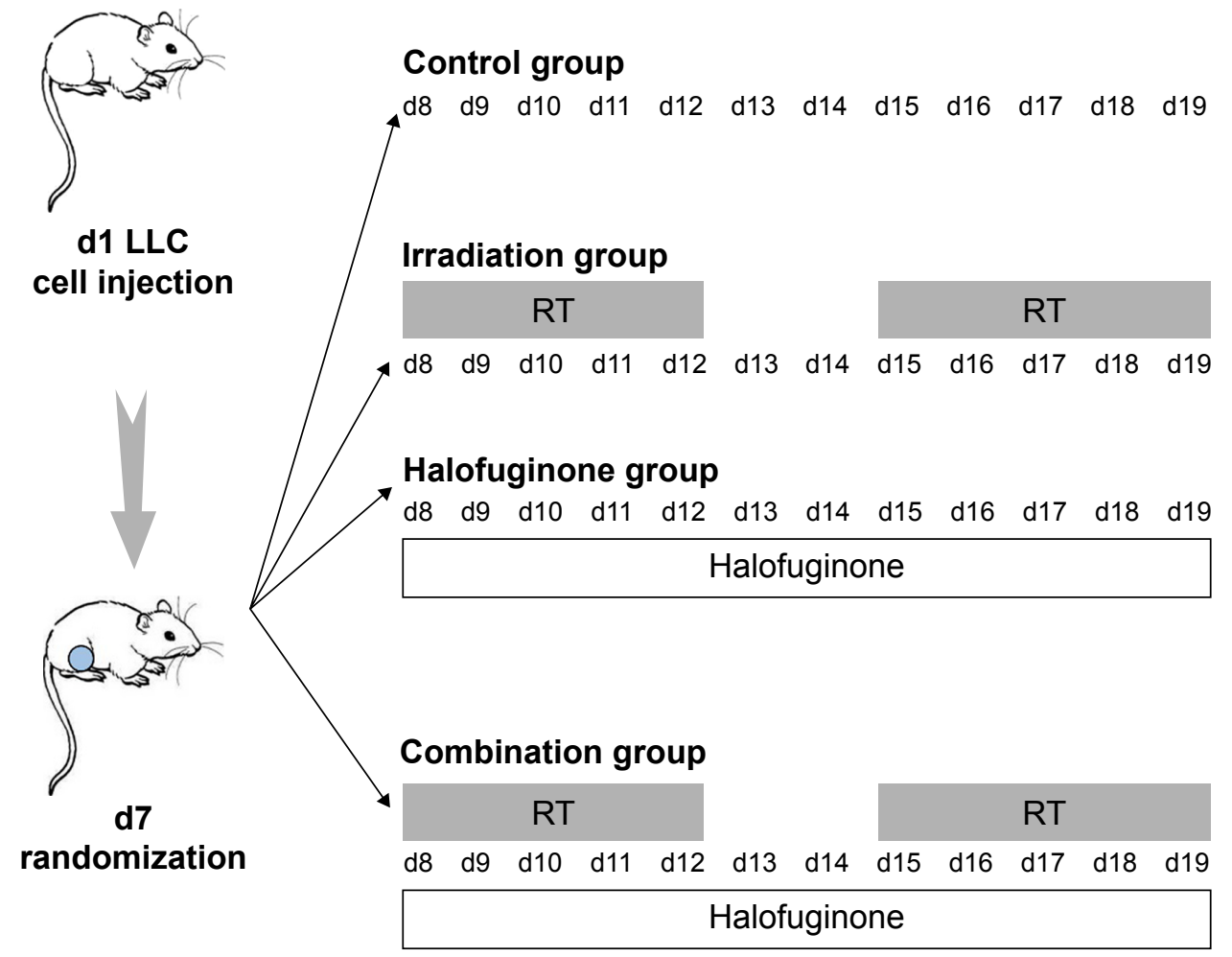

Figure I Schematic of tumor models and treatment schedule.

Notes: Suspensions of LLC cells were subcutaneously injected into the right hind limb of each mouse at a concentration of $6 \times 10^{6}$ cells/injection site. Model animals were randomly divided into the following four groups after the longest diameter of the subcutaneous tumor had attained a size of $0.5 \mathrm{~cm}$ (approximately 7 days): the control group, the irradiation group, the halofuginone group, and the combination group.

Abbreviations: LLC, Lewis lung cancer; RT, radiation therapy. 
added to each well and incubated for 30 minutes at $37^{\circ} \mathrm{C}$ to allow enzyme reaction to occur, and then the cells were washed with PBS five times $\times 3$ minutes. Finally, the reactions were stopped by the stop solution. The absorbances were measured at $450 \mathrm{~nm}$ using a microplate reader, and the results were calculated using a calibration curve prepared from standards.

\section{Western blot analysis}

Following treatment, when $80 \%$ confluence in $25 \mathrm{~cm}^{2}$ flasks was reached, cells were rinsed with PBS and lysed with a radio-immunoprecipitation assay lysis buffer (Santa Cruz Biotechnology Inc., Dallas, TX, USA). Following 30 minutes of incubation on ice, the samples were centrifuged at $14,000 \times g$, and the protein concentration in the supernatant was determined by bicinchoninic acid (BCA) protein assay (Pierce, Rockford, IL, USA). Protein samples were stored at $-80^{\circ} \mathrm{C}$ for subsequent Western blot analysis.

The extracted proteins (equal amounts per lane) were subjected to polyacrylamide gel electrophoresis (PAGE) on $5 \%-10 \%$ Tris-glycine polyacrylamide gel and then transferred to polyvinylidene difluoride (PVDF) membranes (0.45 mm; Millipore, Bedford, MA, USA). After blocking with TBST buffer (20 mM Tris-buffered saline and $0.1 \%$ Tween-20) containing 5\% nonfat dry milk for 1 hour at room temperature, the membranes were incubated with primary antibodies against phosphorylated Smad 2/3 (P-Smad $2 / 3$ ), Smad7, and $\beta$-actin for 2 hours at room temperature or overnight at $4^{\circ} \mathrm{C}$. Membranes were washed three times for 5 minutes each with TBST buffer before incubation with horseradish peroxidase-conjugated secondary antibody (ZS-Bio, Beijing, People's Republic of China) for 60 minutes at room temperature. After washing, the membranes were exposed by using enzyme chemiluminescence, and images were acquired by ChemiDoc XRS (Bio-Rad Laboratories Inc., Hercules, CA, USA). Semiquantification of scanned films was performed using Quantity One-4.6.2 (Bio-Rad).

\section{Scratch assay (wound healing assay)}

The cells were seeded in six-well culture plates (Becton Dickinson and Company, Franklin Lakes, NJ, USA) with a density of $2 \times 10^{6}$ per well and maintained at $37^{\circ} \mathrm{C}$ and $5 \%$ $\mathrm{CO}_{2}$ to permit cell adhesion and the formation of a confluent monolayer. The cell layers were then scored with a sterile $200 \mu \mathrm{L}$ pipette tip to generate one homogeneous wound. After wounding, the cells were washed twice with PBS and incubated with culture medium ( $2 \%$ fetal calf serum) for 8 and 24 hours. Then the images were captured by microscope at $\times 100$ magnifications (Olympus Corporation, Tokyo, Japan), and experiments were carried out in triplicate.

\section{Transwell chamber assay}

Invasive ability of the cells was determined by performing the experiment in 24-well transwell chambers (BD). The polycarbonate filters containing $8 \mathrm{~mm}$ pores were coated on ice with $20 \mu \mathrm{L}$ of Matrigel (Sigma-Aldrich Co., St Louis, MO, USA) at $1 \mathrm{mg} / \mathrm{mL}$. After blocking with $1 \%$ bovine serum albumin (BSA) for 1 hour at $37^{\circ} \mathrm{C}$, the cells $\left(1 \times 10^{5} / \mathrm{mL}\right)$ were suspended in serum-free culture medium, and $200 \mu \mathrm{L}$ of the medium was added to the upper compartments of a transwell chamber. In each lower chamber, $500 \mu \mathrm{L}$ of the medium (10\% FBS) was added. After 48 hours of incubation, the cells from the upper compartment of the filter were removed with a cotton swab, and then the cells on the lower surface of the filter were fixed in ethanol and stained with crystal violet. The cells that invaded through the Matrigel and reached the lower surface of the filter were quantified by counting the number of cells that migrated in eight random microscopic fields per filter at a magnification of $\times 200$ (Olympus Corporation).

\section{Other mechanism studies}

Hematoxylin and eosin stain was used for morphological observations of xenografts and metastatic tumors (in the lungs and liver). Sirius Red was used to stain type I collagen.

\section{Statistical analysis}

The survival period was calculated from the day of inoculation of cancer cells to the day of death of the mouse. Unless otherwise specified, data are expressed as mean \pm standard deviation. Between-group comparisons were performed using chi-square tests, and survival analysis was performed using the Kaplan-Meier method; $P<0.05$ was regarded as statistically significant. Statistical analyses were performed by using Statistical Product and Service Solutions 20.0 software package (IBM Corporation, Armonk, NY, USA).

\section{Results \\ Halofuginone reversed migration and invasion of LLC cells}

Twenty-four hours after irradiation, scratch assay showed increased healing areas and the transwell chamber assay showed increased numbers of transwelled cells compared with the control group. Halofuginone decreased the healing areas and the numbers of transwelled cells compared with the control group. The addition of halofuginone to irradiation decreased the 
healing areas and the numbers of transwelled cells compared with the irradiation-alone group. (Figure 2A, B, and C).

\section{Halofuginone enhances the radiosensitivity of LLC xenografts}

There were no significant differences among the groups prior to treatment. On the fifth day of treatment, a significant reduction in tumor size was observed in the halofuginone group $(P=0.023)$, the irradiation group $(P=0.002)$, and the combination group $(P=0.000)$ compared with the untreated control group; however, no differences were observed among the former three groups. On the ninth day of treatment, average tumor volumes were $175.2 \pm 40.0 \mathrm{~mm}^{3}$ in the control group, $118.5 \pm 13.2 \mathrm{~mm}^{3}$
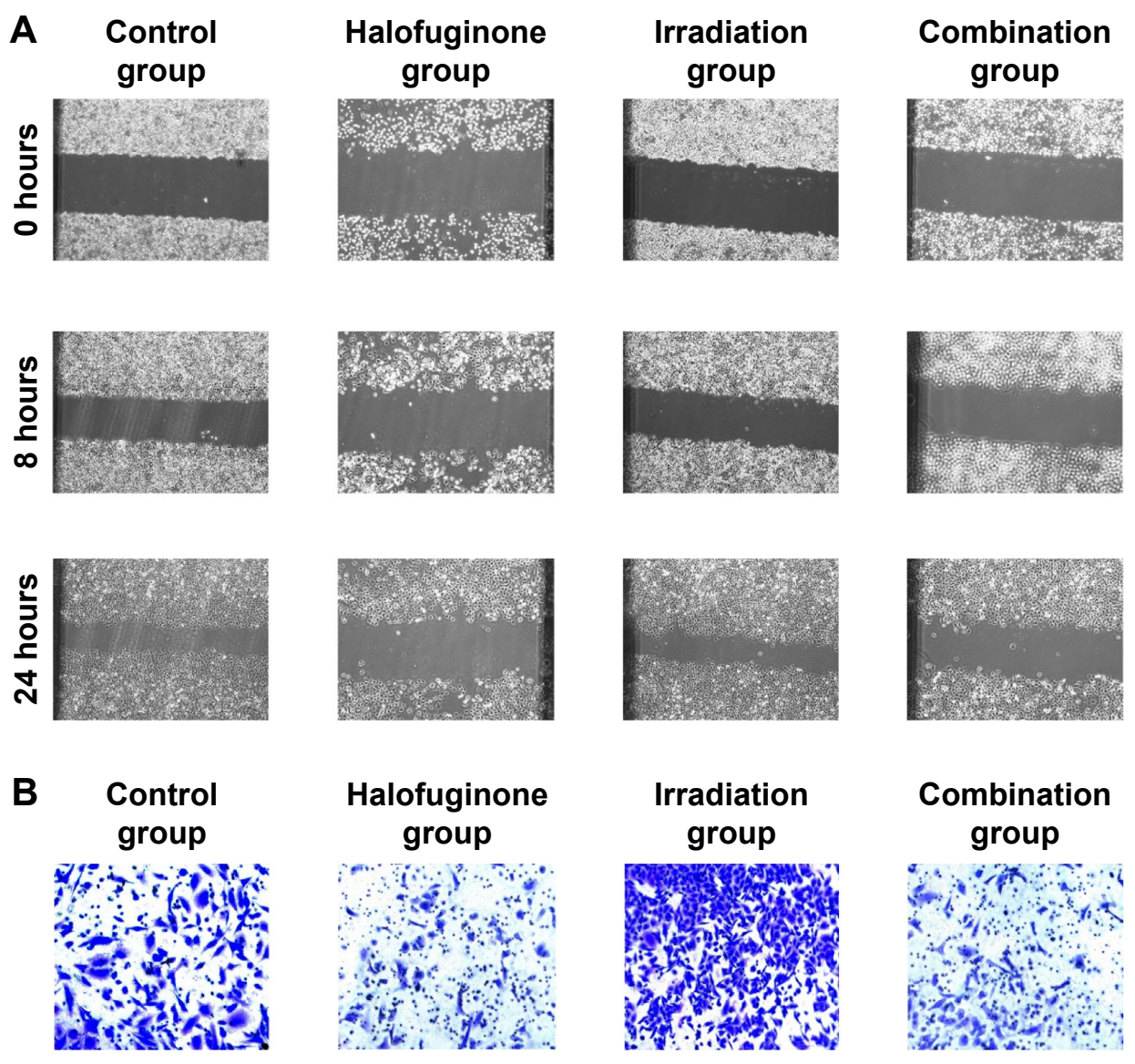

C

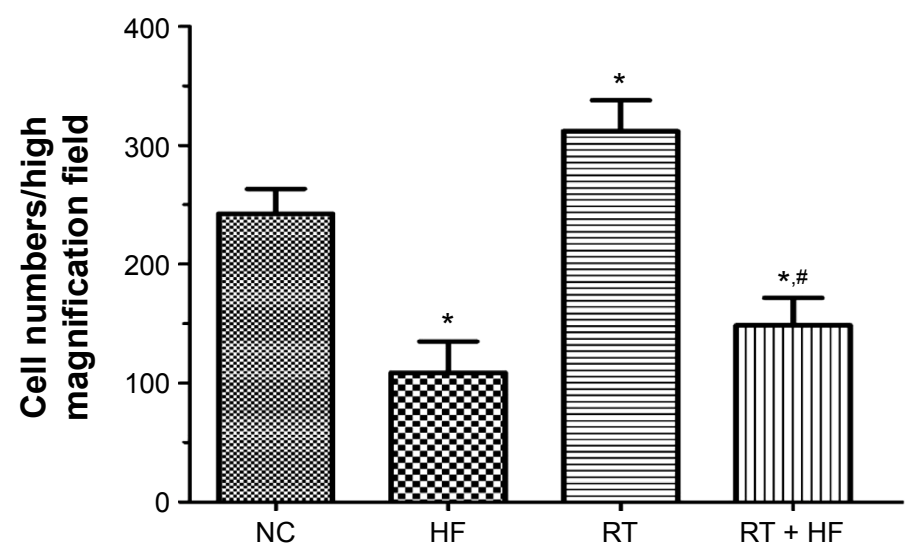

Figure 2 Migration was investigated by scratch assay $(\mathbf{A})$ and invasion was tested by transwell chamber assays (B, C).

Notes: (A) Scratch assay was performed to compare the migratory capabilities of the cells. The images were acquired at $\times 100$ magnification. (B) Transwell chamber assay was used to compare the invasive capabilities of cells. The images were acquired at $\times 200$ magnification. (C) Cell numbers were counted as an average of eight highmagnification fields. *Significant difference $(P<0.05)$ compared with control group. "Significant difference $(P<0.05)$ compared with irradiation group. Abbreviations: NC, negative control; HF, halofuginone; RT, radiation therapy. 
in the halofuginone group, $106.6 \pm 14.0 \mathrm{~mm}^{3}$ in the irradiation group, and $85.6 \pm 17.1 \mathrm{~mm}^{3}$ in the combination group $(P=0.000)$. A significant reduction in the tumor size was observed in the combination group relative to the halofuginone group $(P=0.002)$ and the irradiation group $(P=0.038)$. This phenomenon became increasingly evident as the treatment progressed (Table 1). Figure 3 presents the tumor growth curves for the four groups. Three days after treatment, four mice from each group were randomly euthanized, and their tumors were harvested. At this time, the tumor weights in the control, halofuginone, irradiation, and combination groups were $1.25 \pm 0.17 \mathrm{~g}, 0.72 \pm 0.18 \mathrm{~g}, 0.67 \pm 0.05 \mathrm{~g}$, and $0.44 \pm 0.13 \mathrm{~g}$, respectively $(P=0.000)$; thus, the tumor inhibition rates in the halofuginone, irradiation, and combination groups relative to the control group were $42.4 \%, 46.4 \%$, and $64.8 \%$, respectively. In the combination group, tumor weight was significantly reduced compared with the halofuginone group $(P=0.015)$ and the irradiation group $(P=0.044)$.

\section{The effect of halofuginone on mouse prognoses}

In the control, halofuginone, irradiation, and combination groups, the median survival times were $37.5,65,82.5$, and 87.5 days, respectively $(P=0.002)$. Significant extensions of the survival period were observed in the halofuginone group $(P=0.005)$, the irradiation group $(P=0.016)$, and the combination group $(P=0.001)$ relative to the untreated control group. The longest survival was observed in the combination group, but regrettably, there was no significant difference compared with halofuginone group and irradiation group. Figure 4 presents the survival curves for the four groups of mouse. In addition, the numbers of metastasis sites were greatest in the control group and least in the combination group. The results for pulmonary and hepatic metastases are presented in Table 2.

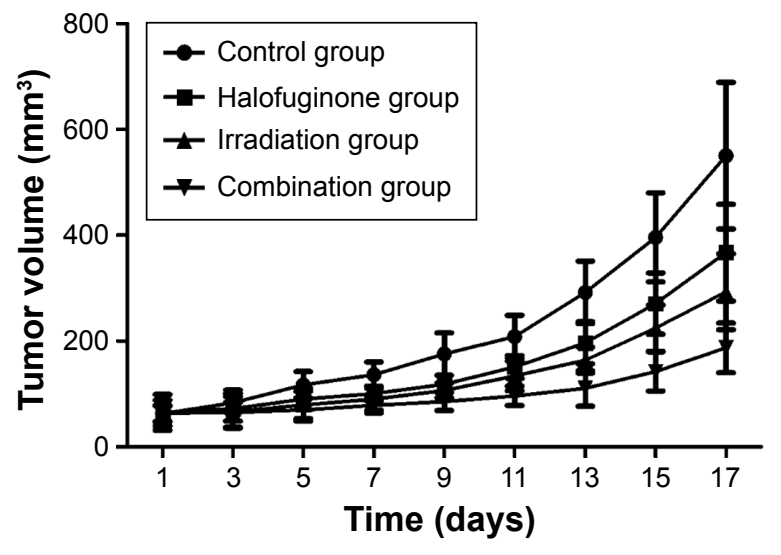

Figure 3 Comparison of four groups in tumor volume.

Note: On the ninth day of treatment, a significant reduction in tumor size was observed in the combination group relative to the halofuginone group $(P=0.002)$ and the irradiation group $(P=0.038)$, and this phenomenon became increasingly evident as the treatment progressed.

\section{The effect of halofuginone on TGF- $\beta$ I levels}

In vivo, both ELISA and IHC results indicated that TGF- $\beta 1$ levels were higher in the irradiation group than in the control group, and there was a statistical difference from IHC score $(P=0.047)$. Halofuginone treatment significantly reduced TGF- $\beta 1$ levels. Levels of TGF- $\beta 1$ in the combination group ( $P<0.05$ for both ELISA and IHC score) and the halofuginone group ( $P<0.05$ for both ELISA and IHC score) were significantly lower than those in the irradiation group and untreated control. But there was no statistical difference between the halofuginone and combination groups (Table 3 and Figure 5).

\section{Halofuginone inhibited activation of TGF- $\beta$ signaling in the process of ionizing radiation on LLC cells}

After irradiation the expression of P-Smad 2/3 in LLC was increased, and the expression of Smad 7 was decreased

Table I Effect of four groups on LLC xenografts in C57BL/6] mice

\begin{tabular}{llll}
\hline Groups & $\begin{array}{l}\text { Time for a twofold increase } \\
\text { in tumor volume (day) }\end{array}$ & $\begin{array}{l}\text { Absolute growth } \\
\text { delay (day) }\end{array}$ & $\begin{array}{l}\text { Normalized } \\
\text { growth delay (day) }\end{array}$ \\
\hline Control group & 5.43 & & \\
Halofuginone group & 6.82 & 1.39 & \\
Irradiation group & 7.66 & 2.23 & 4.15 \\
Combination group & 10.97 & 5.54 & 1.86 \\
\hline
\end{tabular}

Notes: Absolute tumor growth delay caused by treatment is defined as the number of days required by the treated tumors to exhibit a twofold increase in tumor volume from the initiation minus the time in days the untreated tumors required to a twofold increase in tumor volume. Normalized tumor growth delay is defined as the time in days the tumors required to a twofold increase in tumor volume in the mice treated with the combination of halofuginone plus irradiation minus the time in days to reach a twofold increase in tumor volume in mice treated with halofuginone alone. SER: calculated as the ratio of normalized growth delay in mice treated with halofuginone plus irradiation over absolute growth delay in mice treated with irradiation alone.

Abbreviations: LLC, Lewis lung cancer; SER, sensitization enhancement ratio. 


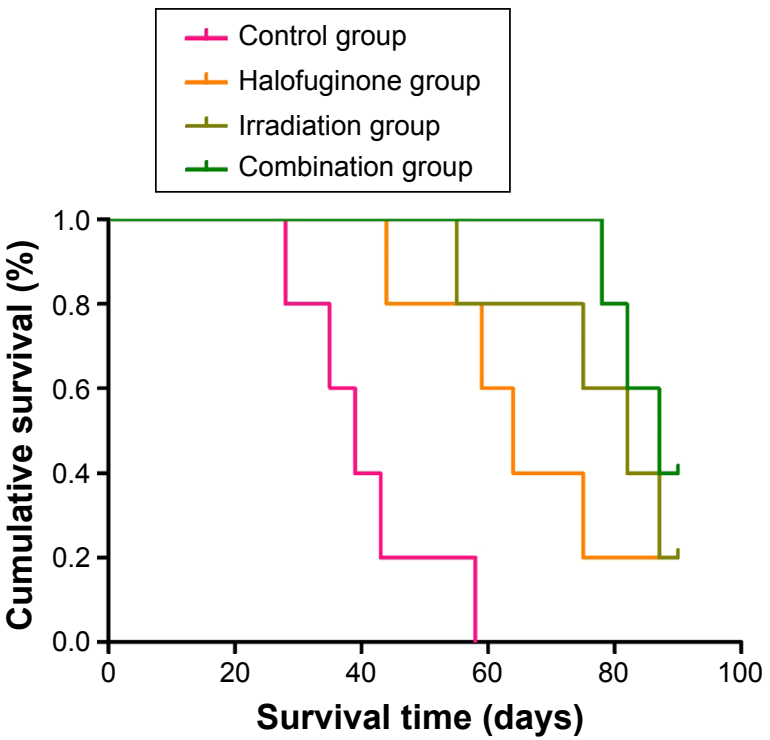

Figure 4 Survival curves of four groups.

Note: The longest survival was observed in the combination group, but regrettably, there was no significant difference when compared with the halofuginone and irradiation groups in the Kaplan-Meier plot of survival for mice.

compared with the control group. Halofuginone treatment could downregulate the expression of P-Smad $2 / 3$ and upregulate Smad7. The addition of halofuginone to ionizing radiation downregulated the $\mathrm{P}$-Smad $2 / 3$ level compared with the irradiation-alone group. Inhibition of Smad7 induced by irradiation was also reversed (Figure 6).

\section{Halofuginone inhibits angiopoiesis and type I collagen expression in LLC xenografts}

There was no significant difference between the MVD counts of the control group and the irradiation group. However, in the combination group, the MVD count was significantly reduced compared with the control group $(P=0.000)$ and the irradiation group $(P=0.000)$ (Table 3 and Figure 7). Sirius Red staining revealed reduced type I collagen expression in the halofuginone and combination groups compared with the control and irradiation groups (Figure 8).

Table 2 Results for pulmonary and hepatic metastases

\begin{tabular}{lll}
\hline Group & $\begin{array}{l}\text { Pulmonary } \\
\text { metastases (n) }\end{array}$ & $\begin{array}{l}\text { Hepatic } \\
\text { metastases (n) }\end{array}$ \\
\hline Control group & 38 & 53 \\
Halofuginone group & 3 & 3 \\
Irradiation group & 9 & 6 \\
Combination group & 2 & 2 \\
\hline
\end{tabular}

Table 3 ELISA, IHC, and MVD results

\begin{tabular}{lllll}
\hline Group & N & $\begin{array}{l}\text { TGF- } \beta \text { I } \\
\text { IHC score }\end{array}$ & $\begin{array}{l}\text { TGF- } \beta \text { I } \\
(\mathbf{p g} / \mathbf{m L})\end{array}$ & $\begin{array}{l}\text { MVD } \\
\text { count }\end{array}$ \\
\hline Control group & 4 & $2.08 \pm 0.27$ & $987.42 \pm 97.58$ & $43.47 \pm 5.03$ \\
Halofuginone group & 4 & $1.32 \pm 0.21$ & $333.31 \pm 47.08$ & $25.40 \pm 3.63$ \\
Irradiation group & 4 & $2.52 \pm 0.3 \mathrm{I}$ & $\mathrm{I}, 120.84 \pm 129.27$ & $38.80 \pm 3.18$ \\
Combination group & 4 & $1.60 \pm 0.24$ & $447.97 \pm 75.92$ & $18.55 \pm 3.07$ \\
\hline
\end{tabular}

Abbreviations: N, number; TGF- $\beta \mathrm{I}$, transforming growth factor-beta I; IHC, immunohistochemistry; MVD, microvessel density; ELISA, enzyme-linked immunosorbent assay.

\section{Discussion}

This study suggested that halofuginone administration can significantly enhance the effects of radiation on LLC cell lines and xenografts. Furthermore, halofuginone treatment can dramatically decrease hepatic and pulmonary metastases. The underlying mechanisms of these phenomena may relate to the inhibitory effects of halofuginone on the TGF- $\beta$ pathway, which affects angiogenesis and the synthesis of type I collagen.

Considerable evidence suggests that the activation of the TGF- $\beta$ pathway is a major contributor to poor prognosis. ${ }^{8,12}$ Reports have indicated that lung cancer patients exhibit elevated TGF- $\beta 1$ levels at baseline and that these levels significantly increase after radiation therapy. ${ }^{7,8,13}$ Plasma TGF- $\beta 1$ levels prior to radiotherapy are correlated with the long-term outcomes of lung carcinoma patients. ${ }^{8}$ In addition, the radiation-induced elevation of circulating TGF- $\beta 1$ during radiation therapy is correlated with non-small-cell lung cancer patient prognosis. ${ }^{12}$ Vujaskovic's findings suggest that elevated TGF- $\beta$ levels during radiotherapy could potentially be indicative of increased risks of developing pulmonary toxicity and experiencing treatment failure ${ }^{14}$ Here, we thought that ionizing radiation can promote the metastatic potential of cancer cells in vitro through TGF- $\beta$-associated events, including angiopoiesis, suppression of immune surveillance, loss of epithelial markers and gain of mesenchymal markers, and degradation of extracellular matrix. In our study, both ELISA and IHC results indicated that TGF- $\beta 1$ levels were highest in the irradiation group; these elevated levels of TGF- $\beta 1$ may negatively affect radiation outcomes in both LLC cells and the xenograft model.

Halofuginone is a plant-derived alkaloid that not only inhibits type I collagen synthesis but also exhibits antifibrotic, antiangiogenic, and antitumor activities. ${ }^{15-19}$ The effects of halofuginone are thought to result from its inhibition of the TGF- $\beta$ signaling pathway. Xavier et al demonstrated that halofuginone induces expression of the TGF- $\beta$ inhibitor Smad7 and decreases TGF- $\beta$ RII levels. ${ }^{20}$ 

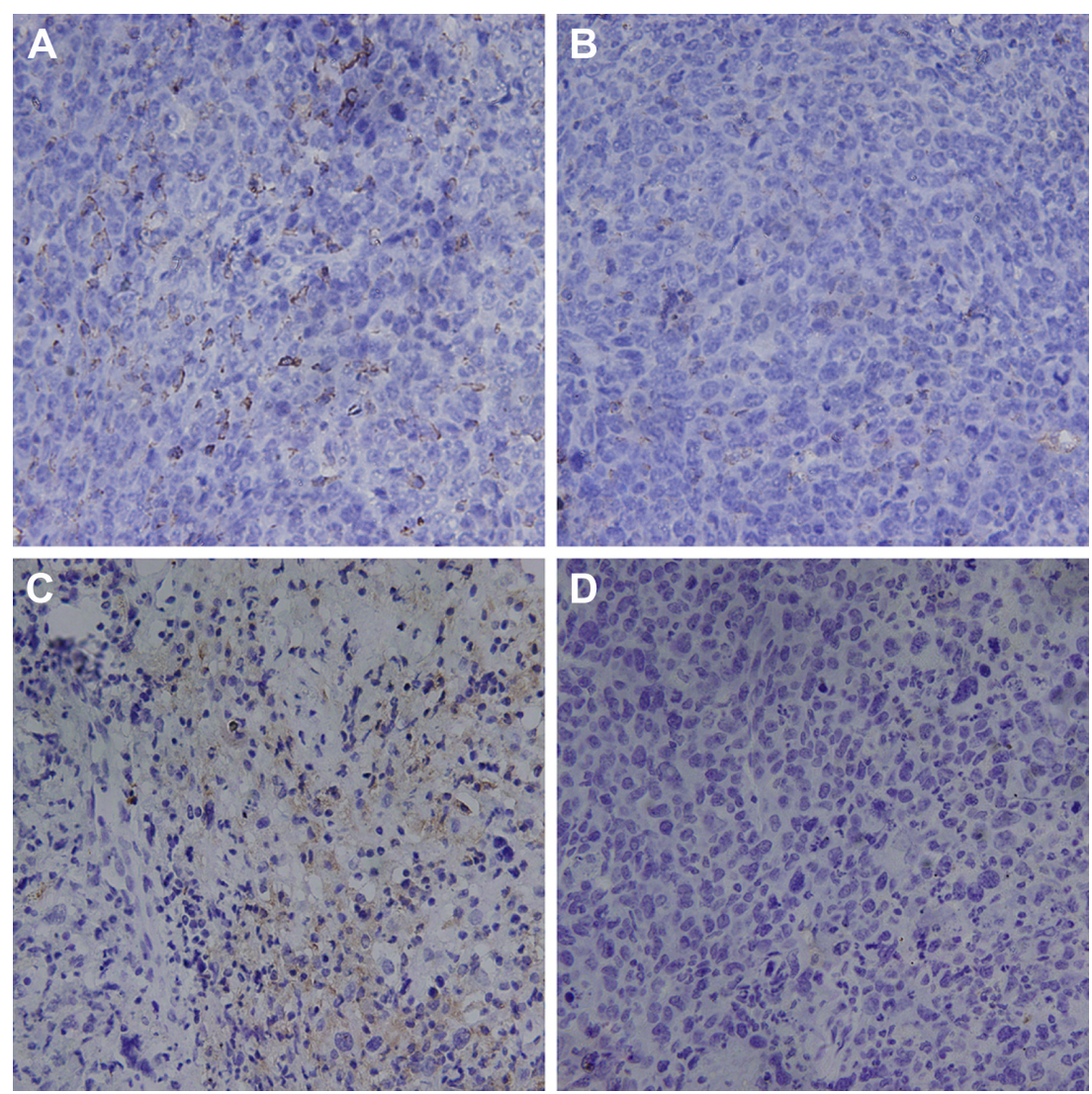

Figure $5 \mathrm{IHC}$ results of TGF- $\beta \mathrm{I}$.

Notes: The expression of TGF- $\beta$ I was located in the cytoplasm or stroma, and after the IHC process, positive area turned into tan color. (A) Presents control group; (B) presents halofuginone group; (C) presents irradiation group; and (D) presents combination group.

Abbreviations: TGF- $\beta$ I, transforming growth factor-beta I; IHC, immunohistochemistry.

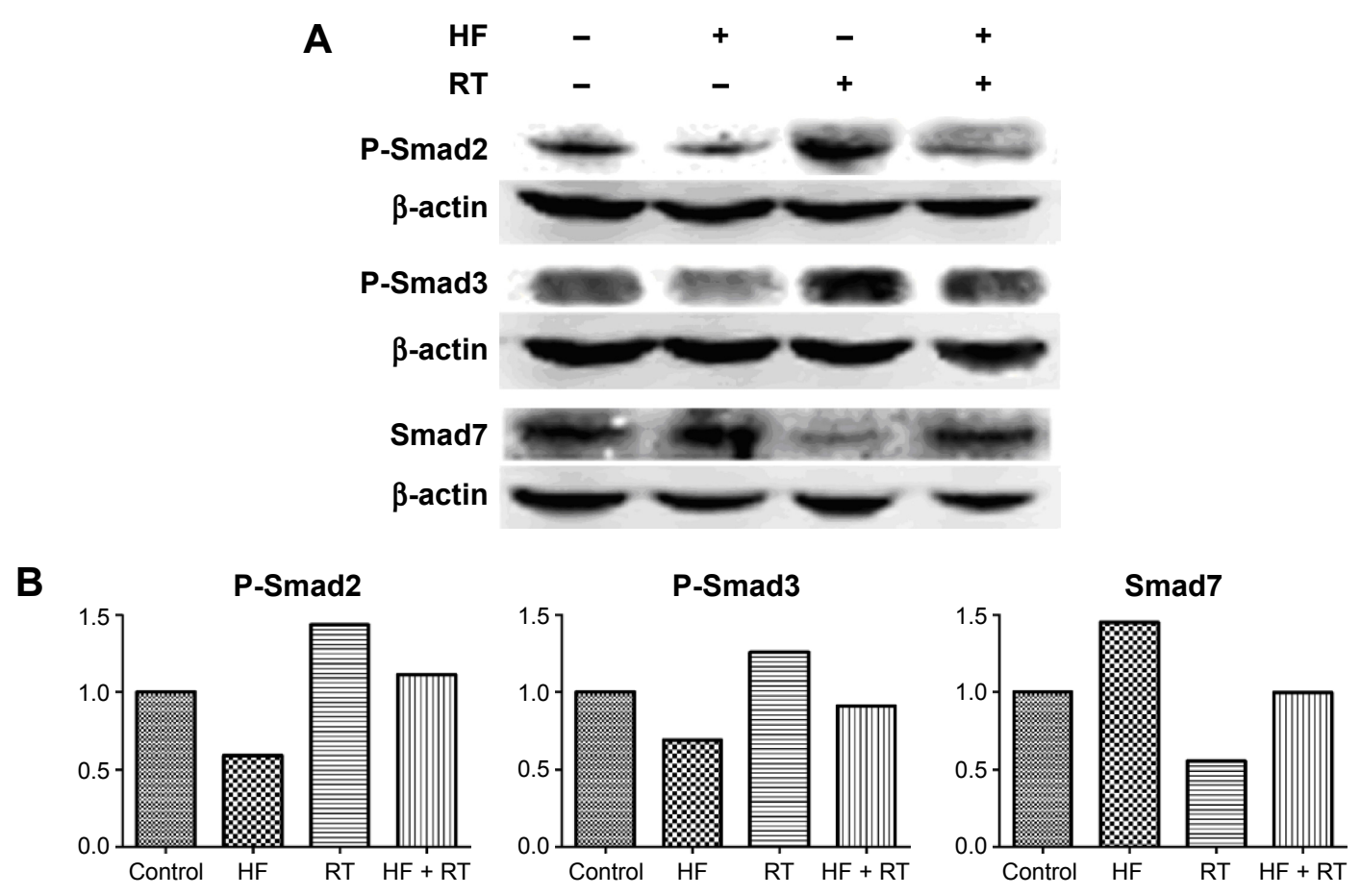

Figure 6 The activation of TGF- $\beta$ signaling pathway in different groups.

Notes: Expression of the downstream contents of TGF- $\beta$ signaling pathway after treatment among different groups, according to Western blot (A). P-Smad $2 / 3$ is an activation marker, while Smad7 is an inhibitor marker of this pathway. Each band from the Western blot was expressed as a percentage vs the level of control group (B). Abbreviations: TGF- $\beta$, transforming growth factor- $\beta$; HF, halofuginone; RT, radiation therapy. 

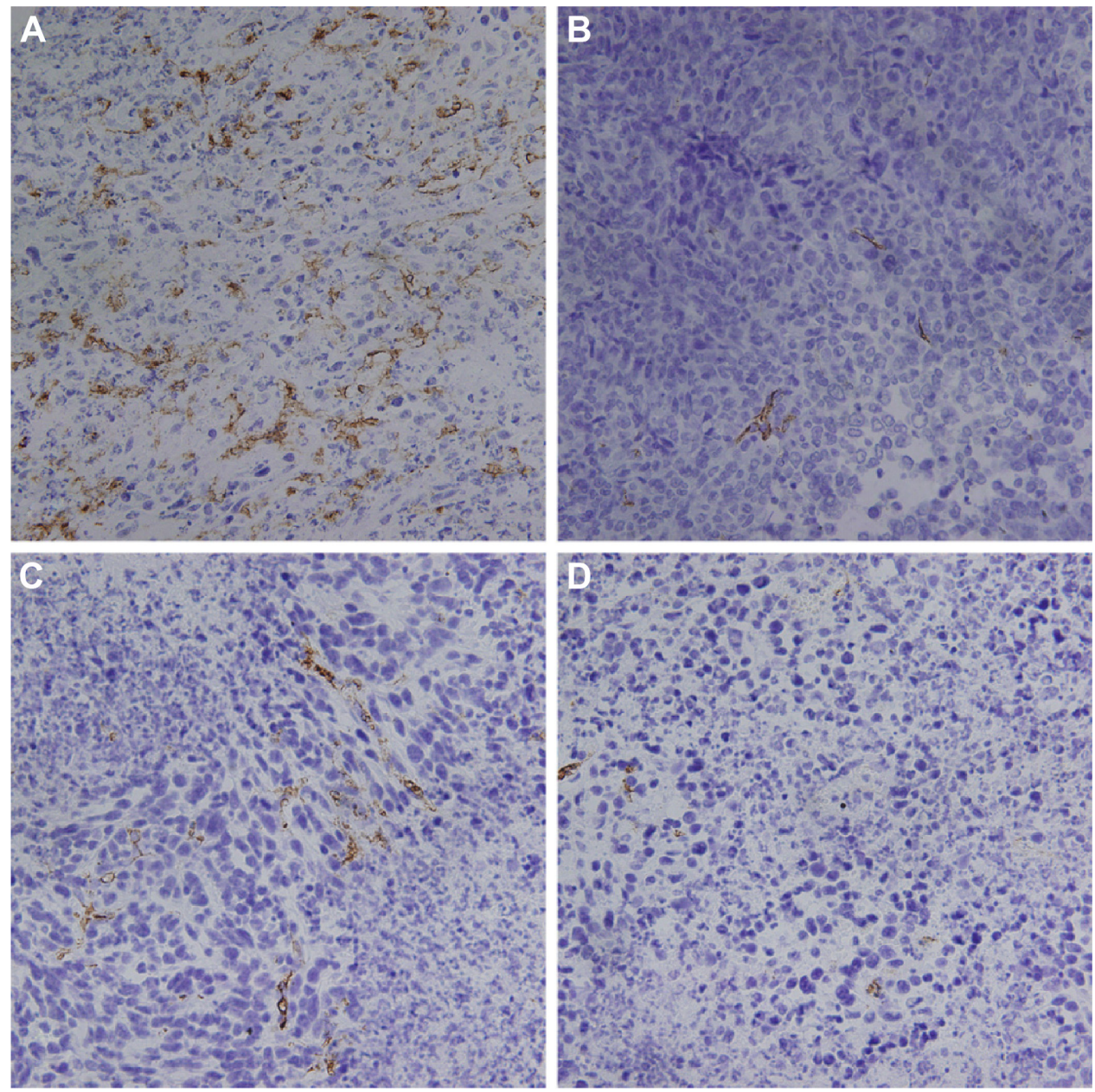

Figure $7 \mathrm{IHC}$ results of CD34.

Notes: CD34 was located in tumor vascular endothelial cell, after treatment of CD34 antibody, After the IHC process, positive areas turned to a tan color. (A) Presents control group; (B) presents halofuginone group; (C) presents irradiation group; and (D) presents combination group.

Abbreviation: IHC, immunohistochemistry.

These effects cause decreased Smad 2 and Smad3 activation and thus inhibit TGF- $\beta$ signaling through the Smad pathway. These findings were consistent with our studies. However, in Xavier's experiment, halofuginone did not affect the therapeutic effect of radiation. Differences in this 2004 study may be because of using: 1) squamous cell carcinoma (SSC) cells rather than LLC cells; and 2) only $5 \times 2$ Gy rather than $10 \times 3$ Gy. Halofuginone enhances the radiosensitivity of pancreatic, prostate, and colon cancer cell lines. ${ }^{20}$ In addition, halofuginone more strongly enhanced the radiosensitivity of cells with a p53 mutation than cells with wild-type p53. ${ }^{21}$ This finding is particularly interesting because it suggests the involvement of $\mathrm{p} 53$ in the TGF- $\beta$ gene response. ${ }^{22}$ Our study indicated that halofuginone can both significantly enhance the antitumor effects of irradiation and suppress the development of hepatic and pulmonary metastases. The underlying mechanisms of these phenomena could relate to the inhibitory effects of halofuginone on the TGF- $\beta$ pathway.
One of the anti-metastasis effects of halofuginone may due to its angiogenesis effect. Angiogenesis is essential for the growth and metastasis of malignancies. ${ }^{23}$ In 2003, researchers reported that halofuginone inhibits tumor progression and that its antitumor effects were associated with decreased tumor angiogenesis. ${ }^{24}$ Halofuginone can also specifically inhibit collagen $\alpha 1$ gene expression, resulting in a decrease in collagen synthesis, and the TGF- $\beta$-induced collagen synthesis. ${ }^{25}$ In addition, the anti-angiogenic activity of halofuginone could relate to its inhibition of matrix metalloproteinases (MMPs), because both collagen synthesis and MMP activity are known to be involved in angiogenesis. ${ }^{26}$ In our study, halofuginone treatment significantly reduced type I collagen expression and MVD counts in the combination treatment group compared with the irradiation-only group; these findings are consistent with the aforementioned literature results. Other mechanisms may also contribute to the antitumor effect of halofuginone, which needs further evaluation in our future study. 

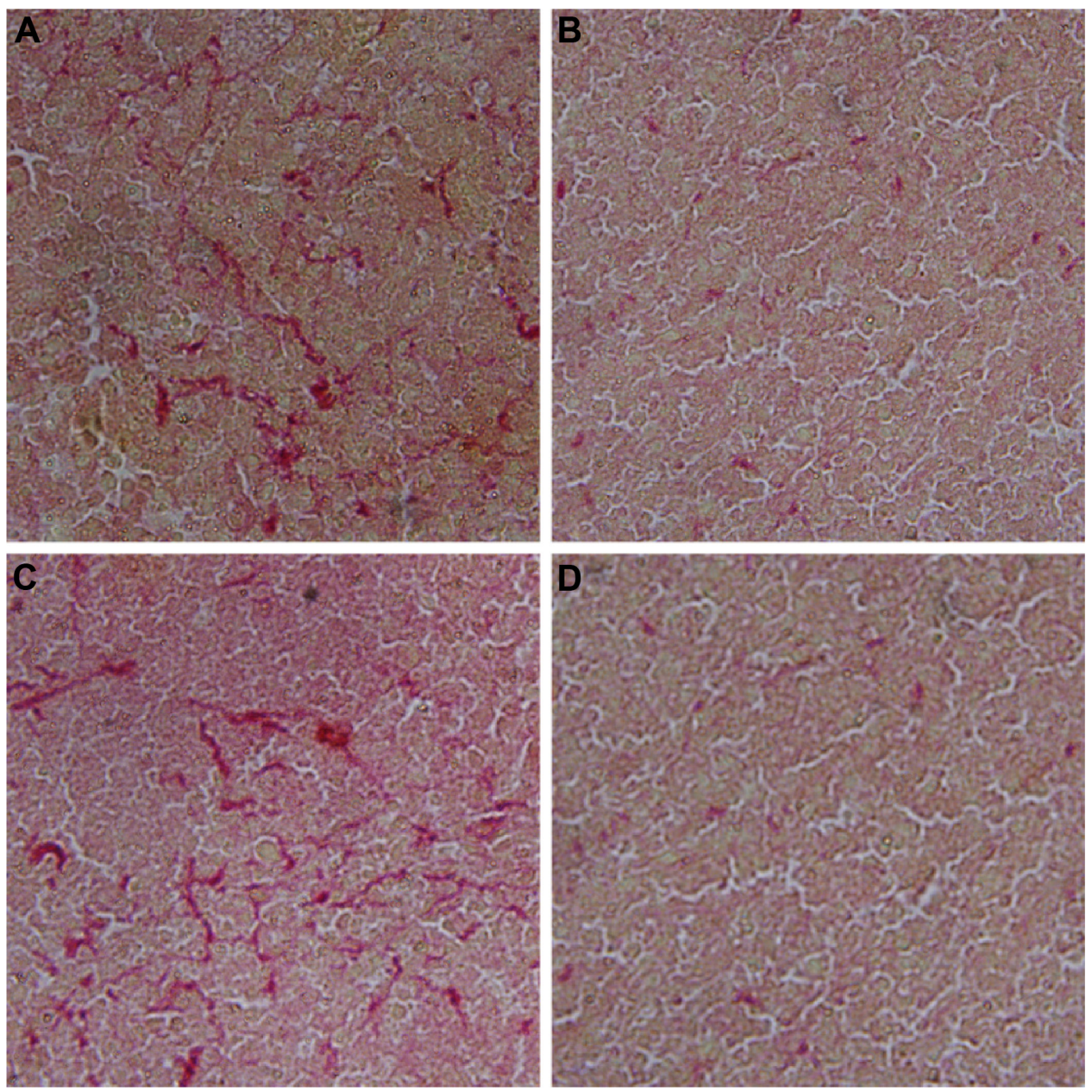

Figure 8 Sirius Red staining for type I collagen.

Notes: Sirius Red, a strong acid anionic dye, reacts with collagen fiber in alkaline perssad. Sirius Red is currently the most significant dye used for staining type I collagen. (A) Presents control group; (B) presents halofuginone group; (C) presents irradiation group; and (D) presents combination group.

\section{Conclusion}

In summary, our study demonstrated that halofuginone has a significant irradiation-sensitizing effect on LLC. Furthermore, the combination of halofuginone and radiation therapy could also inhibit hepatic and pulmonary metastases. The antitumor effects of halofuginone could potentially be attributed to its inhibitory effects on the TGF- $\beta$ signaling pathway, although the detailed mechanisms of halofuginone's antitumor activity warrant additional investigation.

\section{Acknowledgments}

This study was supported by grants from the National Natural Science Foundation of China (No 81372429 and No 30970864). The authors thank American Journal Experts for its linguistic assistance during the preparation of this manuscript.

\section{Disclosure}

No benefits in any form have been or will be received from a commercial party related directly or indirectly to the subject of this paper. The authors report no conflicts of interest in this work.

\section{References}

1. Massague J. TGFbeta in Cancer. Cell. 2008;134(2):215-230.

2. Miyazono K, Ehata S, Koinuma D. Tumor-promoting functions of transforming growth factor-beta in progression of cancer. Ups J Med Sci. 2012; 117(2):143-152.

3. Flavell RA, Sanjabi S, Wrzesinski SH, Licona-Limon P. The polarization of immune cells in the tumour environment by TGFbeta. Nat Rev Immunol. 2010;10(8):554-567.

4. Heldin CH, Vanlandewijck M, Moustakas A. Regulation of EMT by TGFbeta in cancer. FEBS Lett. 2012;586(14):1959-1970.

5. Malaponte G, Zacchia A, Bevelacqua Y, et al. Co-regulated expression of matrix metalloproteinase-2 and transforming growth factor-beta in melanoma development and progression. Oncol Rep. 2010;24(1): 81-87.

6. Ishikura S. Optimal radiotherapy for non-small-cell lung cancer: current progress and future challenges. Gen Thorac Cardiovasc Surg. 2012;60(3): $127-131$.

7. Kong FM, Washington MK, Jirtle RL, Anscher MS. Plasma transforming growth factor-beta 1 reflects disease status in patients with lung cancer after radiotherapy: a possible tumor marker. Lung Cancer. 1996;16(1): $47-59$. 
8. Kong F, Jirtle RL, Huang DH, Clough RW, Anscher MS. Plasma transforming growth factor-betal level before radiotherapy correlates with long term outcome of patients with lung carcinoma. CancerAm Cancer Soc. 1999;86(9):1712-1719.

9. van Kempen LC, Rijntjes J, Mamor-Cornelissen I, et al. Type I collagen expression contributes to angiogenesis and the development of deeply invasive cutaneous melanoma. Int J Cancer. 2008;122(5): 1019-1029.

10. Vanhaesebroeck B, Mareel M, Van Roy F, Grooten J, Fiers W. Expression of the tumor necrosis factor gene in tumor cells correlates with reduced tumorigenicity and reduced invasiveness in vivo. Cancer Res. 1991;51(8):2229-2238.

11. Shimizu M, Saitoh Y, Itoh H. Immunohistochemical staining of Ha-ras oncogene product in normal, benign, and malignant human pancreatic tissues. Hum Pathol. 1990;21(6):607-612.

12. Zhao L, Ji W, Zhang L, et al. Changes of circulating transforming growth factor-beta1 level during radiation therapy are correlated with the prognosis of locally advanced non-small cell lung cancer. J Thorac Oncol. 2010;5(4):521-525.

13. Dancea HC, Shareef MM, Ahmed MM. Role of Radiation-induced TGF-beta Signaling in Cancer Therapy. Mol Cell Pharmacol. 2009;1(1): 44-56.

14. Vujaskovic Z, Groen HJ. TGF-beta, radiation-induced pulmonary injury and lung cancer. Int J Radiat Biol. 2000;76(4):511-516.

15. Yoon YH, Rha KS, Kim DH, Kim EH, Kim JM, Koo BS. Is there any synergic effect for coadministration of mitomycin $\mathrm{C}$ and halofuginone on the skin wound healing? Am J Otolaryngol. 2011;32(2):130-134.

16. Dabak H, Karlidag T, Akpolat N, et al. The effects of methylprednisolone and halofuginone on preventing esophageal and hypopharyngeal fibrosis in delivered radiotherapy. Eur Arch Otorhinolaryngol. 2010;267(9):1429-1435.
17. Spector I, Honig H, Kawada N, Nagler A, Genin O, Pines M. Inhibition of pancreatic stellate cell activation by halofuginone prevents pancreatic xenograft tumor development. Pancreas. 2010;39(7):1008-1015.

18. Nevo Y, Halevy O, Genin O, et al. Fibrosis inhibition and muscle histopathology improvement in laminin-alpha2-deficient mice. Muscle Nerve. 2010;42(2):218-229.

19. Ishii H, Choudhuri R, Mathias A, et al. Halofuginone mediated protection against radiation-induced leg contracture. Int J Oncol. 2009; 35(2):315-319.

20. Xavier S, Piek E, Fujii M, et al. Amelioration of radiation-induced fibrosis: inhibition of transforming growth factor-beta signaling by halofuginone. J Biol Chem. 2004;279(15):15167-15176.

21. Cook JA, Choudhuri R, Degraff W, Gamson J, Mitchell JB. Halofuginone enhances the radiation sensitivity of human tumor cell lines. Cancer Lett. 2010;289(1):119-126.

22. Cordenonsi M, Dupont S, Maretto S, Insinga A, Imbriano C, Piccolo S. Links between tumor suppressors: p53 is required for TGF-beta gene responses by cooperating with Smads. Cell. 2003;113(3):301-314.

23. Carmeliet P, Jain RK. Angiogenesis in cancer and other diseases. Nature. 2000;407(6801):249-257.

24. Gross DJ, Reibstein I, Weiss L, et al. Treatment with halofuginone results in marked growth inhibition of a von Hippel-Lindau pheochromocytoma in vivo. Clin Cancer Res. 2003;9(10 Pt 1):3788-3793.

25. Yavas G, Calik M, Calik G, Yavas C, Ata O, Esme H. The effect of Halofuginone in the amelioration of radiation induced-lung fibrosis. Med Hypotheses. 2013;80(4):357-359.

26. Jordan MC, Zeplin PH. Local inhibition of angiogenesis by halofuginone coated silicone materials. J Mater Sci Mater Med. 2012;23(5): 1203-1210.
OncoTargets and Therapy

\section{Publish your work in this journal}

OncoTargets and Therapy is an international, peer-reviewed, open access journal focusing on the pathological basis of all cancers, potential targets for therapy and treatment protocols employed to improve the management of cancer patients. The journal also focuses on the impact of management programs and new therapeutic agents and protocols on

\section{Dovepress}

patient perspectives such as quality of life, adherence and satisfaction. The manuscript management system is completely online and includes a very quick and fair peer-review system, which is all easy to use. Visit http://www.dovepress.com/testimonials.php to read real quotes from published authors. 